\title{
Sequential medical trials
}

\author{
(stopping rules/asymptotic optimality)
}

\section{T. L. LAI ${ }^{\dagger}$, BRUCE LEVIN $^{\dagger}$, HERBERT ROBBINS ${ }^{\dagger}$, AND DAVID SIEGMUND ${ }^{\ddagger}$ \\ †Department of Mathematical Statistics, Columbia University, New York, New York 10027; and ‡Department of Statistics, Stanford University,} Stanford, California 94305

Contributed by Herbert Robbins, March 24, 1980

\begin{abstract}
A model for sequential clinical trials is discussed. Three proposed stopping rules are studied by the Monte Carlo method for small patient horizons and mathematically for large patient horizons. They are shown to be about equally effective and asymptotically optimal from both Bayesian and frequentist points of view and are markedly superior to any fixed sample size procedure.
\end{abstract}

Consider the design of a clinical trial to select the better of two treatments, A or B, for treating a specified number $N$ of patients. The trial phase involves pairwise allocation of treatments to $n$ pairs of patients, after which the apparently superior treatment is given to the remaining $N-2 n$ patients. We shall assume that the difference in effect of the two treatments is measured by a random variable $z$ which is normally distributed with mean $\delta$ and variance $\sigma^{2}$. If $\delta>0$, treatment $A$ is preferred to treatment $\mathrm{B}$; if $\delta<0$, the preference is reversed; and if $\delta=$ 0 , neither is preferred. The problem is to decide on the number of pairs to be used for the trial phase.

Let $z_{i}$ denote the difference in response between the patient receiving treatment $A$ and the patient receiving treatment $B$ in the $i$ th pair on trial, and let $s_{n}=z_{1}+\ldots+z_{n}$. If $n$ pairs are put on trial and the remaining $N-2 n$ patients are given treatment A or B according to if $s_{n}>0$ or $s_{n}<0$, then the total number of inferior treatments is $n+(N-2 n) I\left(s_{n}<0\right)$ if $\delta>$ 0 and $n+(N-2 n) I\left(s_{n}>0\right)$ if $\delta<0$, where $I(\cdot)$ denotes the indicator function of the event in question. Given a stopping rule $T$ for determining the number of pairs of patients to be put on trial, the regret $R(\delta, T)$ is defined to be the expected total difference in response between the ideal procedure, which would assign all $N$ patients to the superior treatment, and the procedure determined by the stopping rule $T$; hence, $R(\delta, T)$ equals $|\delta|$ times the expected number of inferior treatments, or

$$
\begin{aligned}
R(\delta, T) & =\delta E_{\delta}\left\{T+(N-2 T) I\left(s_{T}<0\right)\right\} \quad \text { if } \quad \delta>0 \\
& =|\delta| E_{\delta}\left\{T+(N-2 T) I\left(s_{T}>0\right\} \quad \text { if } \delta<0 .\right.
\end{aligned}
$$

Our primary purpose is to compare three stopping rules for minimizing Eq. 1 in the case of known $\sigma:(i)$ the Bayes rule $T_{\mathrm{B}}$ for a flat prior on $\delta$; (ii) an ad hoc rule $T_{\mathrm{A}}$ suggested by Anscombe (ref. 1); and (iii) another ad hoc rule $T^{*}$ considered here for the first time. These rules are studied by the Monte Carlo method, and analytic approximations are obtained as $N \rightarrow \infty$. A general conclusion is that all three rules are about equally good from both a frequentist and a Bayesian point of view. We also discuss briefly the case of unknown $\sigma$.

Suppose first that $\sigma$ is known and hence without loss of generality that $\sigma=1$. Let $\Phi$ denote the distribution function and $\phi$ the density function of the standard normal distribution. To provide a standard of comparison for the stopping rules to be

The publication costs of this article were defrayed in part by page charge payment. This article must therefore be hereby marked "advertisement" in accordance with $18 \mathrm{U}$. S. C. $\$ 1734$ solely to indicate this fact. discussed below, suppose for the moment that $|\delta|$ is known and that we need only discover whether $\operatorname{sgn} \delta$ is +1 or -1 . Then a fixed sample size $n$ minimizing $R(|\delta|, n)$ would be obtained by solving the equation

$\frac{\partial}{\partial_{n}} R(|\delta|, n)\left(=|\delta| \frac{\partial}{\partial_{n}}\left\{n+(N-2 n) \Phi\left(-|\delta| n^{1 / 2}\right)\right\}\right)=0$, which defines $n$ implicitly by

$$
g\left(|\delta| n^{1 / 2}\right)=N /(2 n)
$$

where

$$
\begin{aligned}
g(x) & =[2 \Phi(x)-1] /[x \phi(x)]+1 & \text { if } & x>0, \\
& =3 & \text { if } & x=0 .
\end{aligned}
$$

It can be shown that $g(x)$ is increasing in $x \geq 0$. For this minimizing value of $n$, say $n^{*}=n^{*}(|\delta|)$, one can show that for any $\delta \neq 0$

$$
R\left(\delta, n^{*}\right) \sim(2 \log N) /|\delta| \quad \text { as } \quad N \rightarrow \infty .
$$

In practice $|\delta|$ will be unknown, but we can try to estimate it sequentially. Thus, Eq. 2 suggests the following procedure. Stop the trial at stage

$$
T^{*}=\inf \left\{k: g\left(\left|s_{k}\right| / k^{1 / 2}\right) \geq N /(2 k)\right\}
$$

Since $g(x) \geq 3$ for all $x \geq 0, T^{*} \leq N / 6$. Among other properties of $T^{*}$, we shall show that for every fixed $\delta \neq 0, R\left(\delta, T^{*}\right) \sim$ $R\left(\delta, n^{*}\right)$ as $N \rightarrow \infty$. Hence, $T^{*}$ is asymptotically as efficient as the optimal fixed sample size $n^{*}$, which requires knowledge of $|\delta|$.

An alternative approach in ignorance of $|\delta|$ is to assume a prior distribution $G$ on $\delta$ and to choose a stopping rule $T$ to minimize the integrated regret $\int_{-\infty}^{\infty} R(\delta, T) d G(\delta)$. Anscombe (ref. 1) first considered this problem with the flat prior $d G(\delta)$ $=d \delta$. Although the integrated regret with respect to the flat prior is infinite for every stopping rule $T(\geq 1)$, the posterior regret $r(k, s)$ given that $s_{k}=s$ and that stopping occurs at stage $k$ is well defined and can be shown to be

$$
\begin{aligned}
r(k, s)=|s|+k^{-1 / 2} N\left\{\phi\left(s / k^{1 / 2}\right)\right. & \\
& \left.-k^{-1 / 2}|s| \Phi\left(-|s| / k^{1 / 2}\right)\right\} .
\end{aligned}
$$

For fixed $k, r(k, s)$ attains its minimal value at two symmetric points, $s$ and $-s$, defined by the equation

$$
1-\Phi\left(|s| / k^{1 / 2}\right)=k / N \text {. }
$$

Anscombe suggested using Eq. 7 to define a stopping rule

$$
T_{\mathrm{A}}=\inf \left\{k: 1-\Phi\left(\left|s_{k}\right| / k^{1 / 2}\right) \leq k / N\right\},
$$

which, like $T^{*}$, will be shown to be asymptotically optimal from both the Bayesian and the frequentist points of view.

The exact solution $T_{\mathrm{B}}$ to the Bayes problem considered by Anscombe can be computed by the backward induction algorithm (e.g., ref. 2, p. 50). Fig. 1 plots the stopping boundaries of $T^{*}, T_{\mathrm{A}}$, and $T_{\mathrm{B}}$ for $N=100$. 


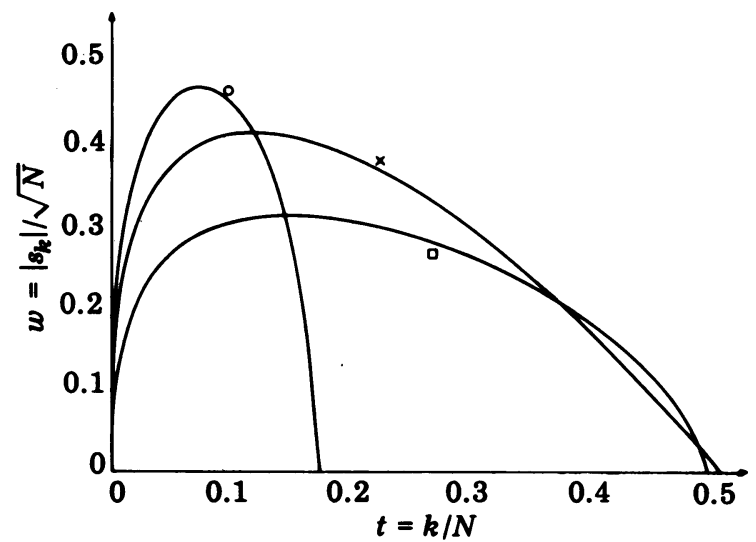

FIG. 1. The boundary $T^{*}(\mathrm{O})$, Anscombe boundary $T_{\mathrm{A}}(\mathrm{X})$, and Bayes boundary $T_{\mathrm{B}}(\square)$ for $N=100$.

\section{An asymptotically optimal class of stopping rules}

Given any continuous function $f:(0,1 / 2] \rightarrow[0, \infty)$, define the stopping rule

$T(f, N)=\inf \left\{k \leq N / 2:\left|s_{k}\right| \geq N^{1 / 2} f(k / N)\right\}(\inf \phi=N / 2)$.

The stopping rule $T^{*}$ in Eq. 5 is of this form with $f(t)$ equal to

$$
f^{*}(t)=t^{1 / 2} g^{-1}(1 / 2 t)
$$

where $g$ is defined in Eq. 3. Likewise Anscombe's rule $T_{A}$ in Eq. 8 is also of this form with $f(t)$ equal to

$$
f_{\mathrm{A}}(t)=t^{1 / 2} \Phi^{-1}(1-t) .
$$

Let $\theta=\delta N^{1 / 2}$. Let $w(t), t \geq 0$, denote the Wiener process with drift coefficient $\theta$ under the probability measure $P_{\delta}$. Let $I_{N}=\{k / N: 1 \leq k \leq N / 2\}$. Under $P_{\delta}$, the sequences $\left\{s_{k}\right\}$ and $\left\{N^{1 / 2} w(k / N)\right\}$ have the same distribution, and therefore the stopping rule $T(f, N)$ has the same distribution as $N \tau_{f, N}$, where $\tau_{f, N}$ is the stopping rule defined on the Wiener process $w(t)$ by

$$
\tau_{f, N}=\inf \left\{t \in I_{N}:|w(t)| \geq f(t)\right\}(\inf \phi=1 / 2) .
$$

As $N \rightarrow \infty, \tau_{f, N}$ converges with probability 1 to

$$
\tau(f)=\inf \left\{t \in\left(0, \frac{1}{2}\right):|w(t)| \geq f(t)\right\} .
$$

The regret $R(\delta ; T(f, N))$ can be expressed in terms of the process $w(t)$ as

$$
R(\delta ; T(f, N))=N^{1 / 2} \rho\left(\theta ; \tau_{f, N}\right),
$$

where we define for any stopping rule $\tau(\leq 1 / 2)$ on $w(t)$

$$
\begin{aligned}
\rho(\theta ; \tau) & =\theta E_{\delta}\{\tau+(1-2 \tau) I(w(\tau) \leq 0)\} \quad \text { if } \quad \theta>0, \\
& =|\theta| E_{\delta}\{\tau+(1-2 \tau) I(w(\tau) \geq 0)\} \quad \text { if } \quad \theta<0 .
\end{aligned}
$$

In connection with the Bayes rule $T_{B}$ with respect to the flat prior on $\delta$, Chernoff and Petkau (3) recently studied the corresponding continuous time problem of minimizing the integrated risk $\int_{-\infty}^{\infty} \rho(\theta ; \tau) d \theta$ among all stopping rules $\tau \leq 1 / 2$ on the process $w(t)$. The minimizing $\tau$ is of the form $\tau\left(f_{B}\right)$ in Eq. 13, and they obtained the following asymptotic expansion of the Bayes optimal boundary $f_{\mathrm{B}}(t)$ as $t \rightarrow 0$ :

$$
f_{\mathrm{B}}(t)=\left\{2 t\left[\log \frac{1}{t}-\frac{1}{2} \log \log \frac{1}{t}-\frac{1}{2} \log 16 \pi+o(1)\right]\right\}^{1 / 2} .
$$

It is interesting to compare Eq. 15 with the following asymptotic expansions of the boundaries $f^{*}(t)$ and $f_{A}(t)$ associated with the rules $T^{*}$ and $T_{\mathrm{A}}$ in Eqs. 5-8: as $t \rightarrow 0$,

$$
\begin{aligned}
& f^{*}(t)=\left\{2 t\left[\log \frac{1}{t}+\frac{1}{2} \log \log \frac{1}{t}-\frac{1}{2} \log 4 \pi+o(1)\right]\right\}^{1 / 2}, \\
& f_{\mathrm{A}}(t)=\left\{2 t\left[\log \frac{1}{t}-\frac{1}{2} \log \log \frac{1}{t}-\frac{1}{2} \log 4 \pi+o(1)\right]\right\}^{1 / 2} .
\end{aligned}
$$

Hence, for small $t$ the three boundaries $f^{*}(t), f_{\mathrm{A}}(t)$, and $f_{\mathrm{B}}(t)$ are close to each other; and $f^{*}(t)>f_{\mathrm{A}}(t)>f_{\mathrm{B}}(t)$, as is illustrated in Fig. 1.

Let $\mathcal{C}$ denote the set of all continuous functions $f:(0,1 / 2] \rightarrow$ $[0, \infty)$ satisfying the following two conditions: $(i)$ as $t \rightarrow 0$,

$$
f(t) \sim\left\{2 t \log \frac{1}{t}\right\}^{1 / 2} ;
$$

and (ii) there exist $\eta<3 / 2$ and $t_{0}>0$ such that for all $0<t \leq$ $t_{0}$,

$$
f(t) \geq\left\{2 t\left(\log \frac{1}{t}-\eta \log \log \frac{1}{t}\right)\right\}^{1 / 2} .
$$

In view of Eqs. 15-17, the boundaries $f^{*}, f_{\mathrm{A}}$, and $f_{\mathrm{B}}$ all belong to $\mathcal{C}$. The following theorem shows that for every given $\delta$, stopping rules of the form $T(f, N)$ in Eq. 9 with $f \in \mathcal{C}$ are asymptotically (as $N \rightarrow \infty$ ) as efficient as the optimal fixed sample size $n *$ which assumes $|\delta|$ known.

THEOREM 1. Let $\mathrm{f} \in \mathcal{C}$. Then for every fixed $\delta \neq 0$,

$$
\mathrm{R}(\delta ; \mathrm{T}(\mathrm{f}, \mathrm{N})) \sim(2 \log \mathrm{N}) /|\delta| \text { as } \mathrm{N} \rightarrow \infty .
$$

Hence, in view of Eq. 4, for every fixed $\delta \neq 0$,

$$
\mathbf{R}(\delta ; \mathbf{T}(\mathbf{f}, \mathrm{N})) \sim \mathbf{R}\left(\delta ; \mathbf{n}^{*}\right) .
$$

Moreover, as $\mathrm{N} \rightarrow \infty$ and $\delta \rightarrow 0$ such that $|\theta|=\mathrm{N}^{1 / 2}|\delta| \rightarrow$ $\infty$,

$$
\mathbf{R}(\delta ; \mathrm{T}(\mathrm{f}, \mathrm{N})) \sim 2\left(\log \theta^{2}\right) /|\delta| \sim \mathbf{R}\left(\delta ; \mathrm{n}^{*}\right) .
$$

The order of magnitude of the regret $R(\delta ; T(f, N))$ given by $[20]$ is asymptotically minimal in the sense of the following theorem.

THEOREM 2. Let a $>1$ and let $\delta_{\mathrm{N}}$ be a sequence of positive constants such that $\delta_{\mathrm{N}} \rightarrow 0$ and $\log \delta_{\mathrm{N}}^{-1}=\mathrm{o}(\log \mathrm{N})$ as $\mathrm{N} \rightarrow \infty$. For every $\mathrm{N} \geq 2$, let $\mathcal{T}_{N}$ denote the class of stopping rules $\mathrm{T}$ $\leq \mathrm{N} / 2$ such that $\mathrm{R}\left(\delta_{\mathrm{N}} ; \mathrm{T}\right) \leq(\log \mathrm{N})^{\mathrm{a}} / \delta_{\mathrm{N}}$. Let $\mathrm{f} \in \mathcal{C}$. Then $\mathrm{T}(\mathrm{f}, \mathrm{N}) \in \mathcal{T}_{\mathrm{N}}$ for all large $\mathrm{N}$ in view of $[21]$, and for every fixed $\delta \neq 0$,

$$
\inf _{\mathrm{T} \in \mathcal{T}_{\mathrm{N}}} \mathrm{R}(\delta ; \mathrm{T}) \sim(2 \log \mathrm{N}) /|\delta| \sim \mathrm{R}(\delta ; \mathrm{T}(\mathrm{f}, \mathrm{N})) \text { as } \mathrm{N} \rightarrow \infty \text {. }
$$

Stopping rules of the form $T(f, N)$ with $f \in \mathcal{C}$ are also asymptotically optimal from a Bayesian point of view. The following theorem shows that these rules are asymptotically Bayes with respect to any prior distribution on $\delta$ that has a positive continuous density in some neighborhood of the origin. Moreover, their integrated regret is of the order of $(\log N)^{2}$ and is therefore much smaller for large $N$ than $\left\{2 N G^{\prime}(0) \times\right.$ $\left.\int|\delta| d G(\delta)\right\}^{1 / 2}$, which can be shown to be asymptotically equivalent to the integrated regret of the Bayes rule using the optimal fixed $\boldsymbol{n}$ for a given prior $\boldsymbol{G}$ on $\delta$.

THEOREM 3. Let $\mathrm{G}$ be a distribution function on $(-\infty, \infty)$ such that $G^{\prime}$ is positive and continuous in some neighborhood of the origin and $\int_{-\infty}^{\infty} /|\delta| \mathrm{dG}(\delta)<\infty$. Let $\mathrm{f} \in \mathcal{C}$. Then as $\mathrm{N} \rightarrow$ $\infty$,

$$
\int_{-\infty}^{\infty} \mathrm{R}(\delta: \mathrm{T}(\mathrm{f}, \mathrm{N})) \mathrm{dG}(\delta) \sim \mathrm{G}^{\prime}(0)(\log \mathrm{N})^{2}
$$

$$
\sim \underset{\mathrm{T}}{\sim \inf } \int_{-\infty}^{\infty} \mathrm{R}(\delta ; \mathrm{T}) \mathrm{dG}(\delta)
$$

The proofs of Theorems 1-3 will be given elsewhere. 
Table 1. Risk of Anscombe's rule for various $N^{a}$

\begin{tabular}{|c|c|c|c|c|c|c|c|c|c|}
\hline \multirow{2}{*}{$\begin{array}{c}\theta= \\
\delta N^{1 / 2}\end{array}$} & \multicolumn{3}{|c|}{$N=400$} & \multicolumn{3}{|c|}{$N=2500$} & \multicolumn{3}{|c|}{$N=10,000$} \\
\hline & $R$ & $P$ & $E$ & $R$ & $P$ & $E$ & $R$ & $\bar{P}$ & $E$ \\
\hline 0 & $v$ & & & 0 & & & v & 0. & \\
\hline 1 & 0.36 & & & 0.37 & & & 0.37 & & \\
\hline 2 & & & & & & & & 0. & \\
\hline 3 & & & & .56 & 0.11 & & .57 & 0.12 & 0. \\
\hline 5 & & 0. & & 0.51 & 0.04 & 0.0 & 0.51 & 0.04 & 0. \\
\hline 10 & & 0.0 & & 0.36 & 0.005 & 0.0 & 0.38 & 0.01 & 0.03 \\
\hline 16 & & 0.0 & 0.02 & 0.27 & 0.001 & 0.02 & 0.29 & 0.003 & 0.02 \\
\hline 24 & 0.28 & $0.4 \cdot 10^{-4}$ & 0.01 & 0.24 & 0.0002 & 0.01 & 0.23 & 0.0007 & 0.01 \\
\hline
\end{tabular}

a $\delta>0, \sigma=1, R=N^{-1 / 2} R(T, \delta), P=P_{\delta}\left\{s_{T}<0\right\}, E=N^{-1} E_{\delta}(T)$.

\section{The case of unknown variance}

In the case of known $\sigma$, writing Anscombe's stopping rule as

$$
T_{\mathrm{A}}=\inf \left\{k: \Phi\left(\frac{\left|s_{k}\right|}{k^{1 / 2} \sigma}\right) \geq 1-\frac{k}{N}\right\},
$$

we can regard $T_{\mathrm{A}}$ as the stopping rule of a symmetric repeated significance test of $H_{0}: \delta<0$ versus $H_{1}: \delta>0$ with nominal significance level at stage $k$ equal to $k / N$. When $\sigma$ is unknown, we can estimate it from the data observed so far, and it is natural to replace $\sigma$ in Eq. 22 by $\hat{\sigma}_{k}$, where

$$
\hat{\sigma}_{k}^{2}=\sum_{i=1}^{k}\left(z_{i}-\bar{z}_{k}\right)^{2} /(k-1), \quad \bar{z}_{k}=s_{k} / k .
$$

For $\delta=0$, while $\Phi$ is the distribution function of $s_{k} /\left(k^{1 / 2} \sigma\right)$ in Eq. 22, $s_{k} /\left(k^{1 / 2} \hat{\sigma}_{k}\right)$ has the $t$ distribution with $k-1$ degrees of freedom. This suggests the following analogue of $T_{\mathrm{A}}$ for the case of unknown $\sigma$ :

$$
\hat{T}=\inf \left\{k \geq 2: F_{k-1}\left(\frac{\left|s_{k}\right|}{k^{1 / 2} \hat{\sigma}_{k}}\right) \geq 1-\frac{k}{N}\right),
$$

where $F_{\nu}$ denotes the distribution function of the $t$ distribution with $\nu$ degrees of freedom. Letting $F_{\nu}(t)=\Phi\left(x_{\nu}(t)\right)$ and

$$
u(t ; \nu)=\left\{\nu \log \left(1+\frac{t^{2}}{\nu}\right)\right\}^{1 / 2},
$$

it is convenient to use in Eq. 23 the following two simple approximations to $x_{\nu}(t)$ due to Wallace (4):

$$
\begin{gathered}
u_{1}(t ; \nu)=u(t ; \nu)\left\{1-\frac{1}{2 \nu}\right\}^{1 / 2} ; \\
u_{2}(t ; \nu)=u(t ; \nu)\left\{1-\frac{2\left(1-\mathrm{e}^{-y^{2}}\right)^{1 / 2}}{8 \nu+3}\right\},
\end{gathered}
$$

with

$$
y=\frac{(0.184)(8 \nu+3)}{\nu^{1 / 2} u(t ; \nu)}
$$

As shown by Wallace, $u(t ; \nu) \geq x_{\nu}(t) \geq u_{1}(t, \nu)$, and $u_{2}(t ; \nu)$ provides an excellent approximation to $x_{\nu}(t)$ over a wide range of values of $\nu$ and $t$. Thus instead of $\hat{T}$, it is more convenient to use the stopping rule

$$
\hat{T}_{i}=\inf \left\{k \geq 2: \Phi\left[u_{i}\left(\left|s_{k}\right| /\left(\hat{\sigma}_{k} k^{1 / 2}\right) ; k-1\right)\right] \cdot \geq 1-k / N\right\}
$$

for $i=1$ or 2 . It may be shown analogously to Theorems 2 and 3 that the stopping rules $\hat{T}, \hat{T}_{1}$, and $\hat{T}_{2}$ are asymptotically optimal as $N \rightarrow \infty$ in the case of unknown $\sigma$, from both the Bayesian and frequentist points of view.

\section{Error probability and expected sample size approximations}

Suppose again that $\sigma=1$. Two of the three terms in the regret defined by $\mathrm{Eq} .1$ are $E_{\delta}(T)$, the expected number of pairs in the trial phase, and $P_{\delta}\left\{s_{T}<0\right\}(\delta>0)$, the probability of making an incorrect decision for the post-trial phase. In sequential hypothesis testing, in the absence of a specific loss function, it is customary to examine these two quantities separately in evaluating a test. Hence, it is of interest to give approximations to $E_{\delta}(T)$ and $P_{\delta}\left(s_{T}<0\right)$ for $\delta>0$.

Suppose that as $t \rightarrow 0$, for some $\eta<3 / 2$ and $c>0$ (see Eqs. 15-17)

$f(t)=\{2 t(\log (1 / t)-\eta \log \log (1 / t)-\log c+o(1))\}^{1 / 2}$

By theorem 3 of ref. 5 , for each $\delta \neq 0$ as $N \rightarrow \infty$

$$
\begin{array}{r}
E_{\delta}\{T(f, N)\}=\delta^{-2}\left\{2 \log \left(N \delta^{2}\right)-2(1+\eta) \log \log \left(N \delta^{2}\right)\right. \\
\left.-\log 4 c^{2}-1+K_{\delta}+o(1)\right\},
\end{array}
$$

where $K_{\delta}$ can be computed numerically and equals 1.166 . . $\delta+\delta^{2} / 4+o\left(\delta^{2}\right)$ as $\delta \rightarrow 0$. However, this result does not yield good numerical approximations, because for $\delta$ near 0 , the factor $\delta^{-2}$ magnifies the effect of the asymptotically negligible $o(1)$ in Eq. 29. Although difficult to justify rigorously, the ideas in ref. 5 suggest that $E_{\delta}\{T(f, N)\}$ should approximately equal the root $x$ of the equation

$$
\begin{aligned}
x=\delta^{-2}\{2 \log (N / x)-2 \eta \log \log (N / x) \\
\left.-\log c^{2}-1+2 / \log \left(N \delta^{2}\right)\right\}+1.166 / \delta .
\end{aligned}
$$

\begin{tabular}{|c|c|c|c|c|c|c|c|c|c|c|c|c|c|c|c|c|c|c|}
\hline \multirow{2}{*}{$\begin{array}{c}\theta= \\
\delta N^{1 / 2}\end{array}$} & \multicolumn{3}{|c|}{$n^{*}$} & \multicolumn{3}{|c|}{$T^{*}$} & \multicolumn{3}{|c|}{$T_{\mathrm{A}}$} & \multicolumn{3}{|c|}{$T_{\mathrm{B}}$} & \multicolumn{3}{|c|}{$\hat{T}_{1}$} & \multicolumn{3}{|c|}{$\hat{T}_{2}$} \\
\hline & $R$ & $P$ & $E$ & $R$ & $P$ & $E$ & $R$ & $P$ & $E$ & $R$ & $P$ & $E$ & $R$ & $P$ & $E$ & $R$ & $P$ & $E$ \\
\hline 0.5 & 0.22 & 0.43 & 0.17 & 0.21 & 0.39 & 0.13 & 0.22 & 0.42 & 0.18 & 0.22 & 0.42 & 0.10 & 0.22 & 0.41 & 0.14 & 0.22 & 0.42 & 0.13 \\
\hline 1 & 0.39 & 0.34 & 0.16 & 0.38 & 0.39 & 0.13 & 0.37 & 0.32 & 0.16 & 0.37 & 0.33 & 0.09 & 0.37 & 0.33 & 0.13 & 0.39 & 0.35 & 0.12 \\
\hline 2 & 0.61 & 0.22 & 0.15 & 0.53 & 0.20 & 0.12 & 0.53 & 0.17 & 0.15 & 0.56 & 0.23 & 0.09 & 0.56 & 0.21 & 0.12 & 0.57 & 0.21 & 0.12 \\
\hline 3 & 0.70 & 0.14 & 0.13 & 0.60 & 0.11 & 0.12 & 0.55 & 0.08 & 0.13 & 0.61 & 0.14 & 0.08 & 0.65 & 0.13 & 0.11 & 0.65 & 0.13 & 0.10 \\
\hline 4 & 0.73 & 0.10 & 0.11 & 0.58 & 0.06 & 0.10 & 0.54 & 0.03 & 0.11 & 0.61 & 0.09 & 0.07 & 0.61 & 0.08 & 0.08 & 0.65 & 0.09 & 0.08 \\
\hline 5 & 0.72 & 0.07 & 0.09 & 0.57 & 0.03 & 0.09 & 0.51 & 0.02 & 0.09 & 0.56 & 0.06 & 0.05 & 0.63 & 0.05 & 0.08 & 0.64 & 0.05 & 0.08 \\
\hline 6 & 0.71 & 0.05 & 0.08 & 0.50 & 0.01 & 0.08 & 0.47 & 0.01 & 0.07 & 0.50 & 0.04 & 0.05 & 0.61 & 0.04 & 0.06 & 0.62 & 0.04 & 0.06 \\
\hline 7 & 0.68 & 0.04 & 0.07 & 0.50 & 0.003 & 0.07 & 0.47 & 0.004 & 0.06 & 0.46 & 0.03 & 0.04 & 0.58 & 0.03 & 0.05 & 0.58 & 0.03 & 0.05 \\
\hline 8 & 0.66 & 0.03 & 0.06 & 0.51 & 0.001 & 0.06 & 0.44 & 0.002 & 0.05 & 0.43 & 0.02 & 0.04 & 0.51 & 0.01 & 0.05 & 0.56 & 0.02 & 0.05 \\
\hline 9 & 0.63 & 0.02 & 0.05 & 0.50 & 0.000 & 0.06 & 0.45 & 0.001 & 0.05 & 0.40 & 0.01 & 0.03 & 0.52 & 0.01 & 0.04 & 0.52 & 0.01 & 0.04 \\
\hline 10 & 0.60 & 0.02 & 0.04 & 0.50 & 0.000 & 0.05 & 0.41 & 0.001 & 0.04 & 0.37 & 0.008 & 0.03 & 0.52 & 0.01 & 0.04 & 0.52 & 0.01 & 0.04 \\
\hline 20 & 0.43 & 0.005 & 0.02 & 0.48 & 0.000 & 0.02 & 0.38 & 0.000 & 0.02 & 0.29 & 0.000 & 0.01 & 0.52 & 0.000 & 0.03 & 0.52 & 0.000 & 0.03 \\
\hline
\end{tabular}

We hope that solving Eq. 30 will provide useful approximations to $E_{\delta}(T)$ when it is not too small and when $P_{\delta}\left\{s_{T}<0\right\}$ is near 0 . Comparisons with the Monte Carlo results in Table 1 indicate that this is the case.

It seems a fairly delicate problem to approximate $\boldsymbol{P}_{\delta}\left\{s_{T}<0\right\}$ for $\delta>0$. However, for Brownian motion and boundaries $f$ satisf ying Eq. 28, a modification of the argument given in ref. 6 shows that as $\theta \rightarrow \infty$

$$
P_{\delta}\{w(\tau(f))<0\} \sim \frac{c(\log \theta)^{\eta-1 / 2}}{2 \pi^{1 / 2} \theta^{2}}
$$

Table 2. Regret, error probability, and expected sample size for $n^{*}, T^{*}, T_{\mathrm{A}}, T_{\mathrm{B}}, \hat{T}_{1}$, and $\hat{T}_{2} \mathrm{a}$

a $N=100, \delta>0, \sigma=1, R=R(\delta, T) / N^{1 / 2}, P=P_{\delta}\left\{s_{T}<0\right\}, E=E_{\delta}(T) / N$. 
This suggests that for $N$ large and $\theta=\delta N^{1 / 2}$ moderately large, and for, say, $f=f_{\mathrm{A}}$,

$$
P_{\delta}\left\{s_{T}<0\right\} \doteq \theta^{-2} .
$$

Comparison with the Monte Carlo results in Table 1 indicate that this is a good approximation for $N=10,000$ and $2 \leq \theta \leq$ 10. For smaller $\theta,[31]$ is not applicable. For larger $\theta$, neglecting the excess over the boundary in using Brownian motion is too crude.

Tables 1 and 2 report the results of two Monte Carlo experiments. Table 2 gives the risk, error probability, and expected sample size of the stopping rules $T^{*}, T_{\mathrm{A}}, T_{\mathrm{B}}, T_{1}$, and $\hat{T}_{2}$ for $\sigma$ $=1$ and $N=100$ and compares these quantities with those of the optimal fixed sample size $n^{*}$, which assumes $|\delta|$ and $\sigma$ known. Table 1 gives similar results for Anscombe's rule and $N=400,2500$, and 10,000 .
This research was supported by the National Institutes of Health, the National Science Foundation, and the Office of Naval Research.

1. Anscombe, F. J. (1963) J. Am. Stat. Assoc. 58, 365-383.

2. Chow, Y. S., Robbins, H. \& Siegmund, D. (1971) Great Expectations: The Theory of Optimal Stopping (Houghton Mifflin, Boston).

3. Chernoff, H. \& Petkau, A. J. (1979) Sequential Medical Trials Involving Paired Data, Technical Report 79-20 (Inst. Appl. Math. Stat., Univ. British Columbia).

4. Wallace, D. L. (1959) Ann. Math. Stat. 30, 1121-1130.

5. Lai, T. L. \& Siegmund, D. (1979) Ann. Stat. 7, 60-76.

6. Lai, T. L. \& Siegmund, D. (1977) Ann. Stat. 5, 946-954. 